\title{
A study of non-motor symptoms in parkinson's disease
}

\author{
Deepali Bhagwan Aendole ${ }^{1^{*}}$, Anita Gaule ${ }^{2}$
}

$\left\{{ }^{1}\right.$ Assistant Professor, Department of Neurology $\}$ \{2Assistant Professor, Department of Physiology $\}$ Topiwala National Medical College \& B. Y. L. Nair Charitable Hospital, Mumbai, Maharashtra, INDIA.

Email: drdeepali1985@yahoo.com

Abstract Background: Parkinson's Disease is reported to be the second most common neurodegenerative pathology of the central nervous system. PD are often under-recognized by treating physicians yet have significant impact on disability and healthrelated quality of life, particularly in advanced disease. NMS leads to severe disability affecting quality of life of patients. Aim and objective: To study the non motor symptoms of Parkinson's disease Methodology: Present study was a cross sectional study carried out in 100 patients with parkinson's disease (PD). Data was collected with pretested questionnaire. Data included demographic data and clinical history. The Hoehn and Yahr (HandY) stage was used to measure disease severity. The non-motor symptom scale (NMSS) was used to assess NMS. Data was analysed with appropriate statistical tests. Results and discussion: Thus the prevalence of NMS was $96 \%$. Most frequent non motor symptom was difficulty in falling asleep (55\%) followed by urinary frequency (42\%) and nocturia (37\%). Most frequently affected domain was sleep $(61 \%)$ followed by urinary domain (59\%). Mean NMSS score in our study was $28.5 \pm 22.16$. Most commonly observed NMS was difficulty in falling asleep (3.5 \pm 4.4$)$ followed by urinary urgency $(3.5 \pm 4.1)$.

Key Word: parkinson's disease.

\section{*Address for Correspondence:}

Dr Deepali Bhagwan Aendole, Assistant Professor, Department of Neurology, Topiwala National Medical College \& B. Y. L. Nair

Charitable Hospital, Mumbai, Maharashtra, INDIA.

Email: drdeepali1985@yahoo.com

Received Date: 04/11/2019 Revised Date: 11/12/2019 Accepted Date: 27/01/2020

DOI: https://doi.org/10.26611/10211522

This work is licensed under a Creative Commons Attribution-NonCommercial 4.0 International License. (cc)) EY-NO

\begin{tabular}{|l|l|}
\hline \multicolumn{2}{|c|}{ Access this article online } \\
\hline Quick Response Code: & Website: \\
& www.medpulse.in \\
& \\
\end{tabular}

\section{INTRODUCTION}

Parkinson's disease (PD), first described by James Parkinson in 1817. ${ }^{1}$ Although Parkinson's disease (PD) has traditionally been considered a motor system disorder, it is now recognized to be a complex disease with diverse clinical features that include nonmotor symptoms (NMS). In general, NMS are divided into 4 domains: neuropsychiatric, sleep, sensory, and autonomic. $^{2}$ According to recent data from the World Health
Organization (WHO), the age-adjusted global incidence ranges from 9.7 to 13.8 per 100,000 population per year. The lowest reported incidence was amongst Asians and African blacks whereas the highest was amongst whites. ${ }^{3}$ The prevalence of the disease can be variable with age, as it's affecting $1 \%$ of the general population above 60 years ${ }^{4}$ and about $4 \%$ in highest age 5 , and it is still a rare disease before the age of 50. ${ }^{6,7}$ Its typical clinical symptoms are resting tremor, cogwheel rigidity, bradykinesia, and postural instability. Men are affected more than females. ${ }^{8}$ PD results from degeneration of the substantia nigra pars compacta and the consequent dysfunction of the dopaminergic nigrostriatal pathway with additional involvement of serotonergic, noradrenergic and cholinergic pathways. The pathogenesis of PD is complex and postulated mechanisms include involvement of ubiquitin proteasome system, mitochondrial dysfunction, oxidative stress and free radical mediated neuronal damage, excitotoxic cell damage, oligodendrocytic interaction and nerve trophic factor depletion. Pathologically, PD is characterized the presence of 
ubiquitinated inclusions, Lewy bodies (LB) and Lewy neurites, which can also occur at a number at a number of extra-nigral locations and involve non-dopaminergic neurotransmitter systems. ${ }^{10} \mathrm{PD}$ presents with motor symptoms and non-motor symptoms (NMS). NMS include autonomic dysfunction (gastrointestinal, genitourinary, cardiovascular, pupillomotor, thermoregulatory and sexual), sleep disturbance (daytime hypersomnolence, nocturnal insomnia, vivid dreaming, and REM sleep behavioural disorder), neuropsychiatric complications (cognitive impairment, depression, anxiety, apathy, impulse control disorders and dopamine dysregulation, visual hallucinations $[\mathrm{VH}]$ and delusional thought disorder), pain and fatigue. NMS can be present even in early PD and can affect quality of life. ${ }^{11,12}$ different questionnaires have been developed to assess the NMS in PD. They include Non Motor Symptoms Questionnaire (NMSQuest) and Non Motor Symptoms Scale (NMSS) ${ }^{13}$, both of which have been extensively used and validated in patients with PD.

Very few studies were conducted in India regarding Non motor symptoms of Parkinson's disease so present study was conducted to observe the NMS in patients of Parkinson's Disease.

Aim and objective: To study the non motor symptoms of Parkinson's disease

\section{MATERIAL AND METHODS}

Present study was a cross sectional study carried out in 100 patients attending Neurology Clinic of a tertiary care hospital from Bombay Hospital, Mumbai. Study population was 60 patients with parkinson's disease (PD). Inclusion criteria: 1. patients with PD who were diagnosed by United Kingdom Parkinson's Disease Brain Bank criteria 2. Patients above 18 years of age 3. Patients willing to participate in the study

Exclusion Criteria: 1. Vascular parkinsonism 2. Drug or toxin induced parkinsonism 3. Patients with dementia with Lewy Bodies 4. Patients with diabetes milletus 5. Patients with Multiple System Atrophy, progressive Supranuclear Palsy and Corticobasal Degeneration.

Study was approved by ethical committee of the institute. A valid written consent was taken from the patients or caretakers of the patient after explaining study to them.

Data was collected with pretested questionnaire. Data included demographic data and clinical history. Detailed history was taken regarding duration of disease, symptoms, treatment taken, associated premorbid condition etc. A through clinical examination was done. All patients underwent routine haematological investigation like complete blood count, renal function tests, random blood sugar etc. The Hoehn and Yahr (HandY) stage was used to measure disease severity. The non-motor symptom scale
(NMSS) was used to assess NMS. The NMSS includes nine domains and a total of 32 questions. The NMSS not only assesses whether or not NMS are present but also rates their frequency (range of 0-4) and severity (range of 0-3). If the product of the frequency and severity was 1 or higher, then 1 point is assigned. The nine domains were the cardiovascular symptoms (two items), sleep/fatigue (four items), mood/cognition (six items), perceptual symptoms/hallucination (three items), attention/memory (three items), gastrointestinal symptoms (three items), urinary symptoms (three items), sexual functions (two items) and miscellaneous (four items). Questions 1-2 were grouped under the cardiovascular/falls symptom subset; questions 3-7 were grouped under the sleep/fatigue symptom subset; questions 8-14 were grouped under the mood symptom subset; questions 15-17 were grouped under the perceptual symptom subset; questions 18-20 were grouped under the cognition symptom subset; questions 21-23 were grouped under the gastrointestinal symptom subset; questions 24-26 were grouped under the urinary symptom subset; questions 27-28 were grouped under the sexual dysfunction symptom subset; and questions 29-32 were grouped under the miscellaneous symptom subset. All data were analyzed using Statistical Package for the Social Sciences software (version 23.0). Descriptive statistics were expressed as means and percentages. Correlation between NMS and HandY stage, duration of disease were assessed using the Pearson's correlation coefficient. A value of $\mathrm{P}<0.05$ was considered statistically significant.

\section{RESULTS}

In our study we studied 100 patients with PD. Mean age of the patients in our study was $61.32 \pm 9.6$ years. Minimum age was 48 years and maximum was 71 years. Mean age of onset was $53.26 \pm 6.01$ years. Out of 100 patients $62 \%$ were male and $46 \%$ were female. Majority of the patients were $(56 \%)$ studied upto $12^{\text {th }}$ class. $44 \%$ were having higher education.

Table 2 showed distribution of patients according to Cohen and Yahr stage. Majority of the patients (45\%) were in the stage 2 followed by stage 3 (27\%). Patients in stage 1 and stage 4 were $24 \%$ and $4 \%$ respectively. The median HandY stage was $2.2 \pm 0.6$. Fig 1 showed frequency of non motor symptoms in patients of Parkinson's disease. Out of 100 patients, 96\% patients had at least one non motor symptoms. Thus the prevalence of NMS was $96 \%$. Most frequent non motor symptom was difficulty in falling asleep (55\%) followed by urinary frequency $(42 \%)$ and nocturia (37\%). Memory impairment and light headedness was observed in $36 \%$ patients. fatiguability was observed in $34 \%$ patients. Least observed NMS was dribbling of saliva $(2 \%)$ and lack of pleasure (4\%) and Hallucination 
(4\%). Thus most frequently affected domain was sleep $(61 \%)$ followed by urinary domain $(59 \%)$. Mood and cognition was affected in $51 \%$ and attention and memory was affected in $41 \%$. Mean NMSS score in our study was $28.5 \pm 22.16$. There was no significant difference in mean NMSS score of male and female $(\mathrm{p}>0.05)$. Fig 2 showed Mean NMS scores in patients of Parkinson's disease. Most commonly observed NMS was difficulty in falling asleep (3.5 \pm 4.4$)$ followed by urinary urgency $(3.5 \pm 4.1)$. Mean

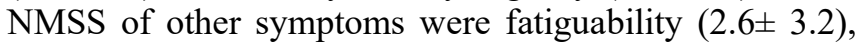

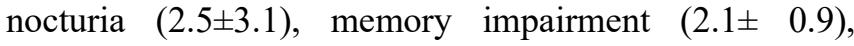

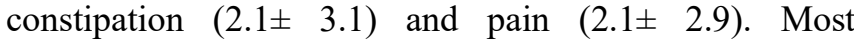
commonly affected domain was sleep with mean NMSS of
$6.34 \pm 5.3$ followed by urinary domain $(6.32 \pm 9.1)$. Mean NMSS of mood /cognition domain was 5.68 \pm 6.7 . Other domains with their mean score were attention/memory (3.74 \pm 5.4$)$, sexual domain (2.11 \pm 3.8$)$, GIT (1.84 \pm 3.1$)$, cardiovascular domain $(1.9 \pm 2.4)$, perceptual problems $(0.84 \pm 2.3)$ and miscellaneous (2.02 \pm 4.1$)$. On analysing correlation of NMSS and duration of disease no significant correlation was found in any domain. Sexual domain was affected more over the duration of disease. On analysing correlation of NMSS with Hand Y stage we found significant correlation in sleep domain (p-0.00), GIT domain (p-0.00), sexual domain (p-0.00) and urinary domain (p-0.03) and severity of the disease. (table 3 )

Table 1: Sociodemographic variables of patients of Parkinson's Disease:

\begin{tabular}{ccc}
\hline Sr no & Variables & Values \\
\hline 1 & Mean age (years) & $61.32 \pm 9.6$ \\
2 & Mean age at onset (years) & $53.26 \pm 6.01$ \\
3 & Duration of disease (years) & $7.34 \pm 1.26$ \\
4 & Sex & \\
5 & Male & $62(62 \%)$ \\
6 & Female & $46(46 \%)$ \\
7 & Level of education \\
8 & Low and middle level & $56(56 \%)$ \\
9 & High level & $44(44 \%)$ \\
\hline
\end{tabular}

Table 2: Distribution of patients of PD according to Cohen and Yahr stage

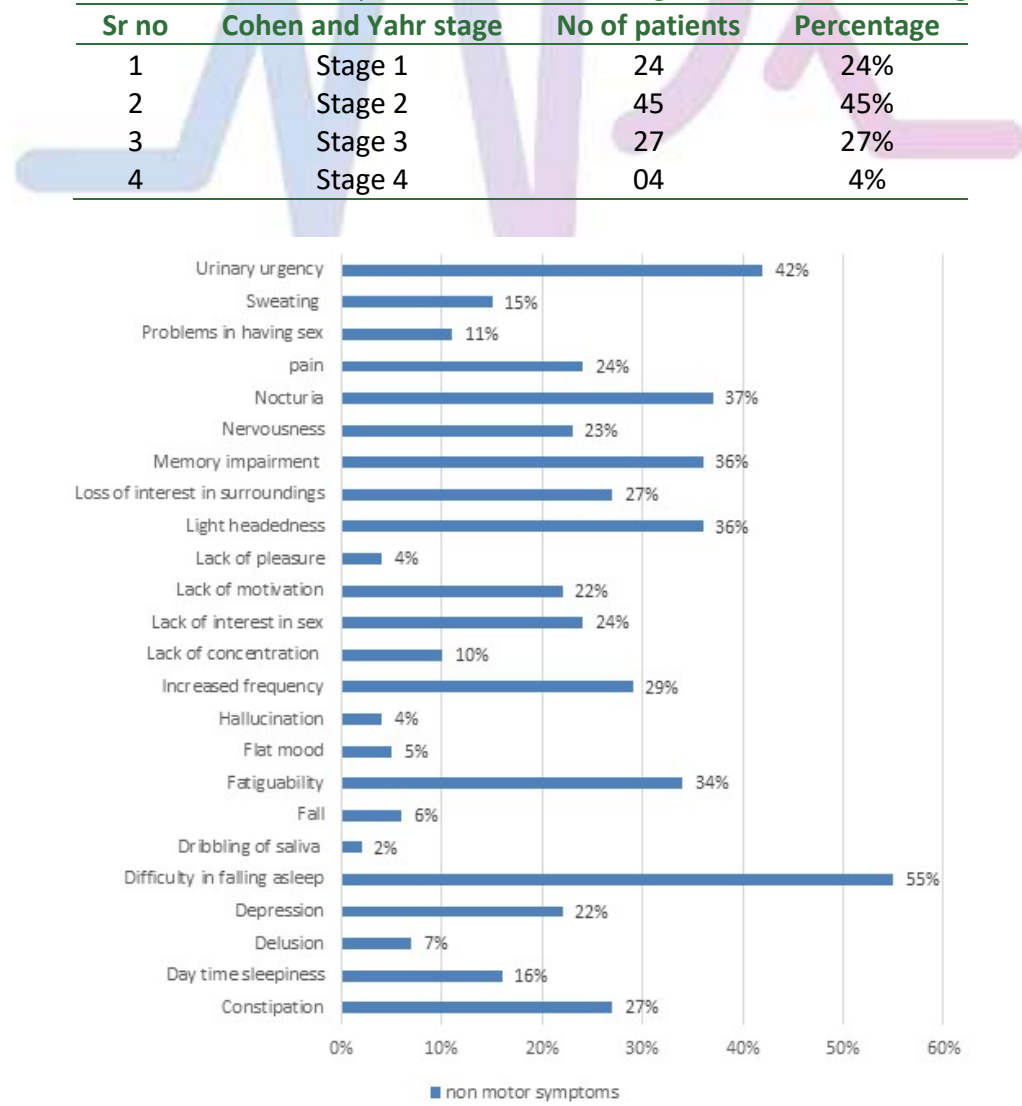

Figure 1: Frequency of non motor symptoms in patients of Parkinson's disease 


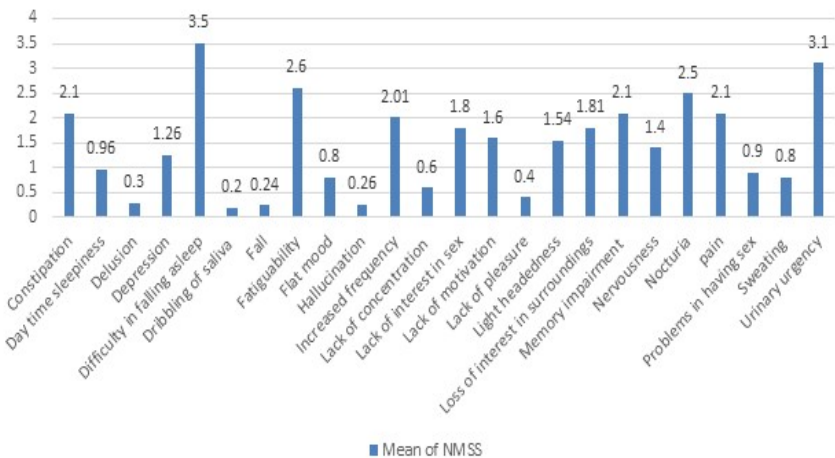

Figure 2: Mean NMSS scores in patients of Parkinson's disease

Table 3: Correlation of NMSS with duration of disease and $\mathrm{H}$ and $\mathrm{Y}$ stage

\begin{tabular}{ccccccc}
\hline Domain & Mean & SD & \multicolumn{2}{c}{ Duration of disease } & \multicolumn{2}{c}{ H andY stage } \\
\cline { 4 - 7 } & & & $\begin{array}{c}\text { Correlation } \\
\text { coefficient }\end{array}$ & P value & $\begin{array}{c}\text { Correlation } \\
\text { coefficient }\end{array}$ & P value \\
\hline Cardiovascular & 1.9 & 2.4 & -0.142 & 0.23 & 0.172 & 0.11 \\
Sleep & 6.34 & 5.3 & -0.081 & 0.51 & 0.462 & $\mathbf{0 . 0 0}$ \\
Mood/cognition & 5.68 & 6.7 & -0.024 & 0.85 & 0.251 & 0.04 \\
Perceptual problems & 0.84 & 2.3 & 0.050 & 0.62 & 0.209 & 0.08 \\
Attention/memory & 3.74 & 5.4 & 0.142 & 0.25 & 0.015 & 0.8 \\
GIT & 1.84 & 3.1 & 0.101 & 0.42 & 0.363 & $\mathbf{0 . 0 0}$ \\
Urinary & 6.32 & 9.1 & 0.123 & 0.33 & 0.261 & $\mathbf{0 . 0 3}$ \\
Sexual & 2.11 & 3.8 & 0.211 & 0.08 & 0.362 & $\mathbf{0 . 0 0}$ \\
Miscellaneous & 2.02 & 4.1 & -0.06 & 0.52 & 0.182 & 0.11 \\
\hline
\end{tabular}

\section{DISCUSSION}

We studied 100 patients with PD for non motor symptoms. Mean age of the patients was $61.32 \pm 9.6$ years. Out of 100 patients $62 \%$ were male and $46 \%$ were female. Preponderance of male was observed in studies like Chandrasekaran $\mathrm{P}$ et al. ${ }^{14}$ and Ravan A et al. ${ }^{15}$ Majority of the patients $(45 \%)$ were in the Cohen and Yahr stage 2 followed by stage 3 (27\%).

In our study, Out of 100 patients, $96 \%$ patients had at least one non motor symptoms. Thus the prevalence of NMS was $96 \%$. Similar findings were observed in P. Barone et al., ${ }^{16} \mathrm{P}$. Martinez-Martin et al. ${ }^{17}$ where prevalence was $98 \%$. In a study by Li et al. ${ }^{18}$, they found an NMS prevalence rate of $100 \%$ in a cohort of 82 patients at a tertiary hospital in China.

In our study, Most frequent non motor symptom was difficulty in falling asleep (55\%) followed by urinary frequency (42\%) and nocturia (37\%). Memory impairment and light headedness was observed in $36 \%$ patients. Thus most frequently affected domain was sleep $(61 \%)$ followed by urinary domain $(59 \%)$. Mood and cognition was affected in $51 \%$ and attention and memory was affected in $41 \%$. Mean NMSS score in our study was $28.5 \pm 22.16$. There was no significant difference in mean NMSS score of male and female ( $>0.05)$. Most commonly observed NMS was difficulty in falling asleep $(3.5 \pm 4.4)$ followed by urinary urgency $(3.5 \pm 4.1)$. Most commonly affected domain was sleep with mean NMSS of $6.34 \pm 5.3$ followed by urinary domain $(6.32 \pm 9.1)$. Similar results were observed in Krishnan S et al., ${ }^{19}$ where sleep was affected in $89 \%$ patients. Similarly, Chaudhuri et al. ${ }^{20}$ sleep domain was most commonly affected in NMS. REM sleep behavioural disorder (RBD) is a parasomnia commonly seen in PD. In a second study, $57 \%$ of patients presenting to a sleep clinic with RBD had an underlying neurological disorder, and of those with PD, 52\% had symptoms of RBD preceding the development of motor symptoms. ${ }^{21} \mathrm{In}$ constract to our study, nocturia, urinary urgency, constipation, and sadness were the most frequently reported NMS in a study by Martinez-Martin P et al. ${ }^{17}$ In study by Shahrul Azmin et al. ${ }^{22}$ the most common reported NMS in the cohort were gastrointestinal symptoms. In a study by Cersosimo et al. $^{23}$, they showed that the gastrointestinal symptoms reported to occur before the onset of motor symptoms were constipation in $87 \%$ of patients, defecatory dysfunction in $58.9 \%$, and dry mouth in $20.5 \%$. Difference in the results of frequent NMS in different studies is due to different study population, different study settings, different drugs used by the patients and different measurement tools. On analysing correlation of NMSS and duration of disease no significant correlation was found in any domain. Sexual domain was affected more over the duration of disease. On analysing correlation of NMSS with Hand Y stage we found significant 
correlation in sleep domain (p-0.00), GIT domain ( $\mathrm{p}-0.00)$, sexual domain (p-0.00) and urinary domain (p-0.03). Similar results were seen in Cosentino et al. ${ }^{24}$ where they observed With advancing HandY stage, the total number of NMS increases.

\section{Acknowledgement: Director of Bombay Hospital.}

\section{REFERENCES}

1. Parkinson J. An essay on the shaking palsy. J Neuropsychiatry Clin Neurosci 2002; 14: 223-36; Discussion, 222

2. S. Lim and A. E. Lang, "The nonmotor symptoms of Parkinson's disease: an overview," Movement Disorders, vol. 25, supplement 1, pp. S123-S130, 2010.

3. Neurological disorders report. Public health challenges.(2016).

http://www.who.int/mental_health/neurology/neurological_di sorders report web.pdf

4. de Lau LM, Breteler MM. Epidemiology of Parkinson's disease. Lancet Neurol (2006) 5(6):525-35.

5. de Rijk MC, Breteler MM, Graveland GA, Ott A, Grobbee DE, van der Meche FG, et al.. Prevalence of Parkinson's disease in the elderly: the Rotterdam study. Neurology (1995) 45(12):2143-6.

6. de Rijk MC, Launer LJ, Berger K, Breteler MM, Dartigues JF, Baldereschi M, et al. Prevalence of Parkinson's disease in Europe: a collaborative study of population-based cohorts. Neurologic diseases in the elderly research group. Neurology (2000) 54(11 Suppl 5):S21-3.

7. Tysnes OB, Storstein A. Epidemiology of Parkinson's disease. J Neural Transm (Vienna) (2017) 124(8):901-5.

8. Tanner CM, Aston DA. Epidemiology of Parkinson's disease and akinetic syndromes. Curr Opin Neurol 2000; 13: 427-30

9. Goetz CG, Fahn S, Martinez-Martin P, Poewe W, Sampaio C, Stebbins GT, Stern MB, Tilley BC, Dodel R, Dubois B, Holloway R, Jankovic J, Kulisevsky J, Lang AE, Lees A, Leurgans S, LeWitt PA, Nyenhuis D, Olanow CW, Rascol O, Schrag A, Teresi JA, Van Hilten JJ, LaPelle N. Movement Disorder Society-sponsored revision of the Unified Parkinson's Disease Rating Scale (MDS-UPDRS): Process, format, and clinimetric testing plan. Mov Disord. 2007;22:417

10. Braak H, Bohl JR, Müller CM, et al.. Stanley Fahn Lecture 2005: The staging procedure for the inclusion body pathology associated with sporadic Parkinson's disease reconsidered. Mov Disord 2006; 21: 2042-51.

11. Khoo TK, Yarnall AJ, Duncan GW, Coleman S, O'Brien JT, Brooks DJ, et al.. The spectrum of nonmotor symptoms in early Parkinson disease. Neurology. 2013 Jan 15;80(3):27681.

12. Duncan GW, Khoo TK, Yarnall AJ, O'Brien JT, Coleman SY, Brooks DJ, et al.. Health-related quality of life in early
Parkinson's disease: The impact of nonmotor symptoms. Mov Disord. 2013 Oct 7

13. P. Martinez-Martin, C. Rodriguez-Blazquez, K. Abe et al.., "International study on the psychometric attributes of the NonMotor Symptoms Scale in Parkinson disease," Neurology, vol. 73, no. 19, pp. 1584-1591, 2009.

14. Chandrasekaran P, Mugundhan K. Non-motor symptoms of Parkinson's disease: Its prevalence across the various stages of Parkinson's disease and its correlation with the severity and duration of the disease. Stanley Med J 2017;4:13-7.

15. Ravan A, Ahmad FM, Chabria S, Gadhari M, Savant Sankhla C. Non-motor symptoms in an Indian cohort of Parkinson's disease patients and correlation of progression of non-motor symptoms with motor worsening. Neurol India 2015;63:16674.

16. P. Barone, A. Antonini, C. Colosimo et al.., "The PRIAMO study: a multicenter assessment of nonmotor symptoms and their impact on quality of life in Parkinson's disease," Movement Disorders, vol. 24, no. 11, pp. 1641-1649, 2009.

17. P. Martinez-Martin, A. H. V. Schapira, F. Stocchi et al.., "Prevalence of nonmotor symptoms in Parkinson's disease in an international setting; study using nonmotor symptoms questionnaire in 545 patients," Movement Disorders, vol. 22, no. 11, pp. 1623-1629, 2007.

18. H. Li, M. Zhang, L. Chen et al.., "Nonmotor symptoms are independently associated with impaired health-related quality of life in Chinese patients with Parkinson's disease," Movement Disorders, vol. 25, no. 16, pp. 2740-2746, 2010.

19. Krishnan S, Sarma MAG, Sarma S, Kishore A. Do non-motor symptoms in Parkinson's disease differ from normal aging? Mov Disord 2011;26:2110-13.

20. Chaudhuri KR, Martinez-Martin P, Brown RG, Sethi K. The metric properties of a novel non-motor symptoms scale for Parkinson's disease: Results from an international pilot study. Mov Disord 2007;22:1901-11.

21. Olson EJ, Boeve BF, Silber MH. Rapid eye movement sleep behaviour disorder: demographic, clinical and laboratory findings in 93 cases. Brain 2000; 123: 331-9.

22. Shahrul Azmin, Abdul Manaf Khairul Anuar, Hui Jan Tan, Wan Yahya Nafisah, Azman Ali Raymond, Othman Hanita, Shamsul Azhar Shah, and Mohamed Ibrahim Norlinah. Nonmotor Symptoms in a Malaysian Parkinson's Disease Population. Hindawi Publishing Corporation Parkinson's Disease Volume 2014, Article ID 472157,7.

23. Cersosimo MG, Raina GB, Pecci C, Pellene A, Calandra CR, Gutiérrez C, et al.. Gastrointestinal manifestations in Parkinson's disease: prevalence and occurrence before motor symptoms. J Neurol (2013) 260(5):1332-8.

24. Cosentino C, Nuñez Y, Torres L: Frequency of non-motor symptoms in Peruvian patients with Parkinson's disease [Article in English, Spanish]. Arq Neuropsiquiat. 2013, $71: 216-21$.

\section{Source of Support: None Declared
Conflict of Interest: None Declared}

Policy for Articles with Open Access:

Authors who publish with MedPulse International Journal of Medicine, Print ISSN: 2550-7583, Online ISSN: 2636-4751 agree to the following terms: Authors retain copyright and grant the journal right of first publication with the work simultaneously licensed under a Creative Commons Attribution License that allows others to share the work with an acknowledgement of the work's authorship and initial publication in this journal.

Authors are permitted and encouraged to post links to their work online (e.g., in institutional repositories or on their website) prior to and during the submission process, as it can lead to productive exchanges, as well as earlier and greater citation of published work. 